\title{
https://doi.org/10.46813/2021-134-074 \\ SPATIO-TEMPORAL STRUCTURE OF CHERENKOV RADIATION EXCITED BY A LASER PULSE IN AN IONIC DIELECTRIC WAVEGUIDE
}

\author{
V.A. Balakirev, P.I. Markov, I.N. Onishchenko \\ National Science Center "Kharkov Institute of Physics and Technology", Kharkiv, Ukraine \\ E-mail: onish@kipt.kharkov.ua
}

The process of excitation of Cherenkov electromagnetic radiation by a laser pulse in an ionic dielectric waveguide, is investigated. The frequency spectrum and mode composition of the Cherenkov radiation are determined. The spatio-temporal structure of the Cherenkov electromagnetic field is obtained and investigated.

PACS: 41.75.Ht, 41.75.Lx, 41.75.Jv, 96.50.Pw

\section{INTRODUCTION}

A laser pulse propagating in a dielectric can excite Cherenkov radiation [ $1-6]$. For the appearance of Cherenkov radiation of a laser pulse, it is necessary that the group velocity of the laser pulse (wave packet) exceeds the phase velocity of an electromagnetic wave propagating in the medium. The effect of Cherenkov radiation of a laser pulse in a dielectric medium is as follows. When a laser pulse propagates in a dielectric, a pulsed ponderomotive force, quadratic in the laser field, will act on the bound electrons of the atoms (ions) of the medium, which, in turn, will lead to polarization of the dielectric. The induced polarization charges and currents will coherently radiate electromagnetic waves. The effect of Cherenkov radiation of a laser pulse is quite equivalent to the Cherenkov radiation of an electron bunch, with the only difference that the ponderomotive force of a laser pulse plays the role of a pulsed electric field of an electron bunch.

In present work, the space-time pattern of the electromagnetic field of Cherenkov radiation, excited by a laser pulse in an ionic dielectric waveguide, is investigated. The frequency spectrum and mode composition of the Cherenkov radiation are determined.

\section{STATEMENT OF THE PROBLEM AND BASIC EQUATIONS}

Let us consider a dielectric waveguide made in the form of a uniform dielectric cylinder of radius, the side surface of which is covered with a perfectly conductive metal film. A circularly polarized laser pulse with electric field components propagates along the waveguide axis

$$
\begin{gathered}
E_{0 x}=\sqrt{\frac{I_{0}}{2}} \psi(r, t), E_{0 y}=i E_{0 x}, \\
\psi=\left[R\left(r / r_{L}\right) T\left(\tau / t_{L}\right)\right]^{1 / 2} .
\end{gathered}
$$

The function $R\left(r / r_{L}\right)$ describes the radial profile of the laser pulse intensity, $r_{L}$ is the characteristic pulse radius $I_{0}=\left|\vec{E}_{0}\right|^{2}, \quad R(0)=1, R(r=b)=0, \quad b$ is the waveguide radius, $T\left(\tau / t_{L}\right)$ is the function, which describes the longitudinal profile, $T\left(\tau / t_{L}\right)=T\left(-\tau / t_{L}\right)$, $\tau=t-z / v_{g}, \quad v_{g}$ is the group velocity of the wave packet, $t_{L}$ is the characteristic duration of the laser pulse, $I_{0}$ is the maximum intensity. The system of Maxwell equations, which describes the electromagnetic field excited by the polarization induced by a laser pulse, has the form

$$
\begin{aligned}
& \operatorname{rot} \vec{E}=-\frac{1}{c} \frac{\partial \vec{H}}{\partial t}, \operatorname{rot} \vec{H}=\frac{1}{c} \frac{\partial \notin \vec{E}}{\partial t}+\frac{4 \pi}{c} \vec{j}_{p o l}, \\
& \operatorname{div} \notin \vec{E}=-4 \pi \operatorname{div} \vec{P}_{N L}, \operatorname{div} \vec{H}=0,
\end{aligned}
$$

where $\hat{\varepsilon}$ is the operator of the permittivity of the ionic dielectric

$$
\vec{j}_{p o l}=\frac{\partial \vec{P}_{N L}}{\partial t}
$$

is the current density of nonlinear polarization, $\vec{P}_{N L}$ is the vector of nonlinear electric polarization, quadratic in the laser field. The nonlinear polarization in an ionic dielectric, induced by the ponderomotive force from the side of the laser pulse, was determined and presented in $[6,7]$.

In Maxwell's equations (2), the nonlinear polarization current is a source of Cherenkov electromagnetic radiation. It is convenient to solve the inhomogeneous system of Maxwell's equations by the Fourier transform method and represent the components of the electromagnetic field in the form of a Fourier integral. For example

$$
E_{z}(r, z, t)=\int_{-\infty}^{\infty} E_{z \omega}(r, z,) e^{-i \omega t} d \omega .
$$

The equation for the longitudinal Fourier component of the electric field has the form

$$
\Delta E_{z \omega}+k_{0}^{2} \varepsilon(\omega) E_{z \omega}=4 \pi\left(\frac{1}{\varepsilon(\omega)} \frac{\partial \rho_{p o l \omega}}{\partial z}-i \frac{k_{0}}{c} j_{z p o l \omega}\right),
$$

where $\rho_{\text {polw }}$ is the Fourier component of the polarization charge density, $j_{z p o l \omega}$ is the Fourier component of the polarization longitudinal current density, $\varepsilon(\omega)$ is permittivity.

For a circularly polarized laser pulse (3), expressions

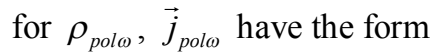

$$
\begin{aligned}
& \rho_{\text {pol } \omega}=\mu\left[\left(\Delta_{\perp}-k_{g}^{2}\right) I_{\omega}(r)+\frac{\varepsilon_{o p t}-1}{6} \Delta_{\perp} I_{\omega}(r)\right] e^{i k_{g} z}, \\
& j_{z p o l \omega}=-\omega k_{g} \mu I_{\omega}(r) e^{i k_{g} z},
\end{aligned}
$$


where $k_{g}=\omega / v_{g}, \Delta_{\perp}$ is the transverse part of the Laplacian, $I_{\omega}(r)$ is the Fourier component of the intensity of the laser pulse field. The parameter $\mu$ is defined in $[5,6]$.

The longitudinal Fourier component of the electric field must satisfy the boundary condition on the side surface of the waveguide

$$
E_{z \omega}(r=b, z)=0 \text {. }
$$

Below we will restrict ourselves to the study of wake fields in the terahertz and infrared ranges. The expression for the permittivity in these ranges can be represented as [7 - 9]

$$
\varepsilon(\omega)=\varepsilon_{\text {opt }} \frac{\omega^{2}-\omega_{L}^{2}}{\omega^{2}-\omega_{T}^{2}}
$$

$\omega_{L}$ is frequency of longitudinal optical phonons, $\omega_{T}$ is frequency of transverse optical phonons, $\varepsilon_{o p t}$ is permittivity in the optical frequency range.

Equation (4) together with boundary condition (5) makes it possible to find the longitudinal Fourier component of the electric field. Calculation the Fourier Integral (3), in turn, gives possible to determine the total field excited by a laser pulse in a dielectric waveguide.

The total field contains the field of longitudinal oscillations, and the field of transverse $\left(\operatorname{div} \vec{E}_{t}=0\right)$ electromagnetic waves. The frequency spectrum of longitudinal vibrations contains only the frequency of longitudinal optical phonons $\omega_{L}$. Behind the laser pulse, the longitudinal field of optical phonons has the form of a monochromatic wave

$$
E_{l z}(r, \tau)=E_{L} \Phi(r) \widehat{T}\left(\Omega_{L}\right) \cos \omega_{L} \tau, \quad \Omega_{L}=\omega_{L} t_{L},
$$

$E_{L}$ is amplitude of the oscillations. The function $\Phi(r)$ describes the distribution of the wake potential in the radial direction. For the Gaussian model of the longitudinal profile of the laser pulse intensity

$$
T\left(t_{0} / t_{L}\right)=\exp \left(-t_{0}^{2} / t_{L}^{2}\right),
$$

we have

$$
\AA\left(\Omega_{L}\right)=\sqrt{\pi} \exp \left(-\Omega_{L}^{2} / 4\right) .
$$

Longitudinal optical phonons are most efficiently radiated when the coherence condition $\omega_{L i} t_{L} \leq 1$ is satisfied. If this condition is not satisfied, longitudinal optical phonons are emitted incoherently, and the wave amplitude is exponentially small.

Let us present expressions for the amplitudes of longitudinal optical phonons for two ionic dielectrics: sodium chloride $\mathrm{NaCl}$ and potassium iodide $\mathrm{KI}$. These two alkali halide ionic crystals $\mathrm{NaCl}$ and $\mathrm{KI}$ are chosen due to the relatively high polarizability of ions.

The obtained expressions for the amplitude of excited longitudinal optical phonons are the followings: for $\mathrm{NaCl}$

for KI

$$
E_{L}(\mathrm{NaCl})=7.4 \cdot 10^{4} \frac{N_{L}}{\lambda_{L}[\mathrm{Mm}]} a_{0}^{2}[\mathrm{~V} / \mathrm{cm}],
$$

$$
E_{L}(K I)=1.16 \cdot 10^{4} \frac{N_{L}}{\lambda_{L}[\mathrm{Mm}]} a_{0}^{2}[\mathrm{~V} / \mathrm{cm}] .
$$

Here $N_{L}$ is the number of wavelengths in the laser pulse, $f_{L}=7.62 \cdot 10^{12} \mathrm{~Hz}$ for $\mathrm{KI}$ and $f_{L}=4 \cdot 10^{12} \mathrm{~Hz}$ for $\mathrm{NaCl}$ are the frequencies of longitudinal optical phonons, $a_{0}=\frac{e E_{0}}{m c \omega_{L}}$ is the parameter of the wave force, $\omega_{L}$ is the frequency of the laser pulse.

Let us now consider the excitation of wake electromagnetic waves by a laser pulse in ionic dielectric waveguide. Behind the laser pulse, the Cherenkov electromagnetic field is a superposition of the radial harmonics of the dielectric waveguide. For the bi-Gaussian profile of the laser pulse intensity (7) and

$$
R\left(r / r_{L}\right)=\exp \left(-r^{2} / r_{L}^{2}\right) .
$$

The expression for the Cherenkov electromagnetic field has the form

$$
\begin{gathered}
E_{t z}(r, \tau)=-E_{w} \sqrt{\pi} \Sigma(r, \tau), \\
\Sigma(r, \tau)=\sum_{n=1}^{\infty} E_{n} J_{0}\left(\lambda_{n} \rho\right) \cos \left(\omega_{n c h} \tau\right), \\
E_{n}=\kappa_{n}^{2}{\Theta_{n}}^{-\frac{\Omega_{n c h}^{2}}{4}} \frac{\lambda_{n}^{2}}{J_{1}^{2}\left(\lambda_{n}\right)}, \\
{\oint_{n}}^{1 / \eta_{L}} \int_{0}^{-\rho^{2}} J_{0}\left(\lambda_{n} \eta_{L} \rho\right) \rho d \rho, \\
\eta_{L}=r_{L} / b, \Omega_{n c h}=\omega_{n c h} t_{L}, \\
\omega_{n c h}=\frac{c}{b} \frac{\lambda_{n}}{\sqrt{\varepsilon_{s t}-\varepsilon_{o p t}}} \kappa_{n},
\end{gathered}
$$

$\omega_{n c h}-$ are the frequencies of the excited radial modes of the dielectric waveguide, which are in the terahertz (infrared) range $\omega_{n c h}<\omega_{T}$,

$$
\kappa_{n}=\frac{1}{\sqrt{1+\omega_{n d}^{2} / \omega_{T}^{2}}}
$$

$\varepsilon_{s t}=\varepsilon_{o p t} \frac{\omega_{L}^{2}}{\omega_{T}^{2}}$ is static dielectric constant, $J_{0}(x)$ is Bessel function, $\lambda_{n}$ are roots of this function.

For $\mathrm{NaCl}$ and $\mathrm{KI}$ ion dielectric waveguide the excited amplitude can be represented in a form convenient for numerical estimates

$$
\begin{gathered}
E_{w}(\mathrm{NaCl})=1.62 \cdot 10^{9} \frac{W(J)}{\{b[\mathrm{Mm}]\}^{4}}[\mathrm{~V} / \mathrm{cm}], \\
E_{w}(K I)=3.45 \cdot 10^{9} \frac{W(J)}{\{b[\mathrm{Mm}]\}^{4}}[\mathrm{~V} / \mathrm{cm}],
\end{gathered}
$$

where $W(J)$ is the laser pulse energy in joules.

\section{ANALYSIS OF NUMERICAL RESULTS}

Fig. 1 shows the values of the frequencies of the radial modes of the Cherenkov field of a laser pulse in a dielectric waveguide with a radius $b=100 \mathrm{~mm}$ for the above crystals. With an increase in the number of the radial mode $n$, in accordance with relation (11), the frequency first increases and then reaches the frequency of transverse optical phonons $\omega_{T}$. 


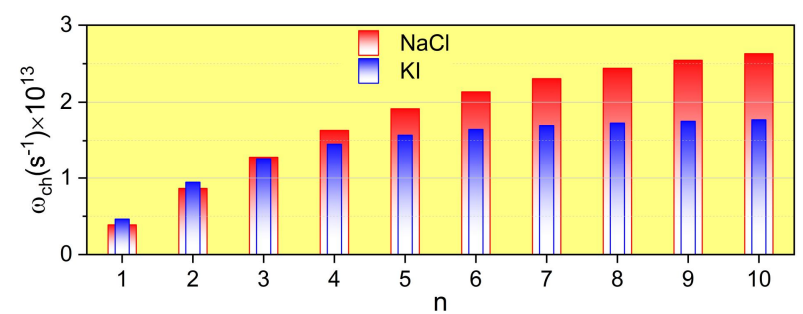

Fig. 1. Dependencies of the radial mode frequency on its number $n$. Nacl-red, KJ-blue

The distribution of the wake field in the dielectric waveguide behind the laser pulse is described by function (9). In Fig. 2 the dependences of this function on the variable $\tau=\left(t-z / v_{g}\right) c / b$ are shown, which is obtained for a laser pulse with a wavelength $\lambda_{L}=1 \mathrm{Mm}$ and duration $t_{L}=20 \mathrm{fs}$. This figure illustrates the case of a laser pulse with a relatively small laser pulse radius $r_{L}=0.1 b$. As follows from the figure, the distribution of the wakefield in the longitudinal direction at a fixed instant of time (or the dependence of the wake field on time at a given point behind the laser pulse) has a complicated non-periodic character.

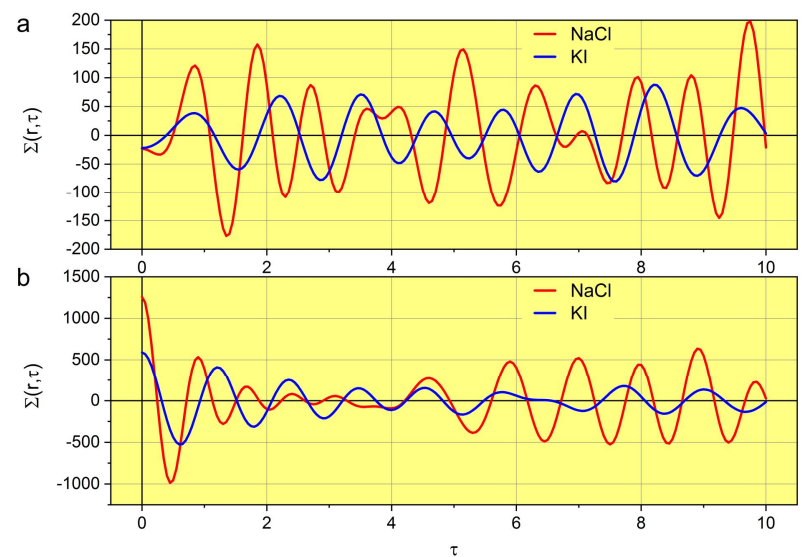

Fig. 2. Dependencies $\Sigma(r, \tau)$ for $r=0.4 b(a)$ and on the axis $r=0(b), r_{L}=0.1 b$

In Fig. 3 the dependences of the amplitude of the radial mode $E_{n}$ (10) of the Cherenkov field on the number $n$ are shown. From this dependence it follows that in the case of laser pulse with a small radius $r_{L}=0.1 b$, a multimode Cherenkov excitation regime is realized. Moreover, the maximum amplitude is for the radial mode with $n=5$ for $\mathrm{NaCl}$ crystal and with $n=4$ for KI crystal.

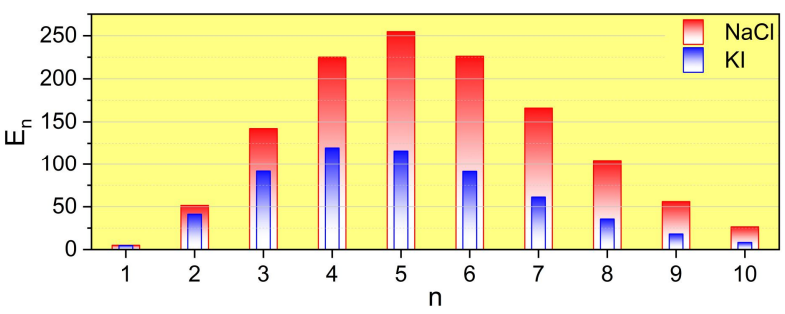

Fig. 3. Dependencies of the amplitude of the radial mode $E_{n}$ of the Cherenkov field on the numbern,

$$
r_{L}=0.1 b
$$

With an increase in the radius of the laser pulse, there is a significant change in the pattern of the wakefield excitation by a laser pulse in an ionic dielectric. Figs. 4 and 5 show similar dependences obtained for the radius of the laser pulse $r_{L}=0.3 b$. The field, as before, has a non-periodic character. It can be seen that the maximum in the distribution on the amplitudes of the radial modes is shifted to the region of lower numbers. For both considered crystals, the maximum falls on the second radial mode, which has much lower frequency (see Fig. 1).

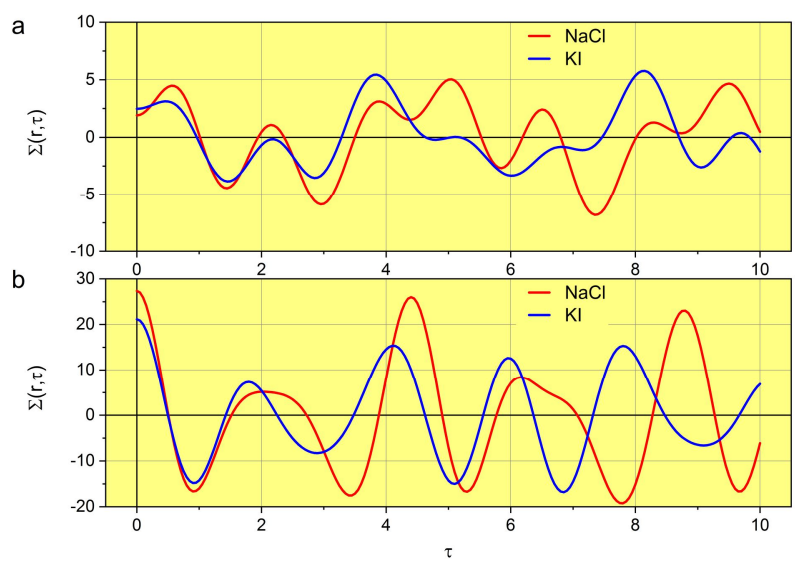

Fig. 4. Dependencies $\Sigma(r, \tau)$ for $r=0.4 b(a)$ and on the axis $r=0(b), r_{L}=0.3 b$

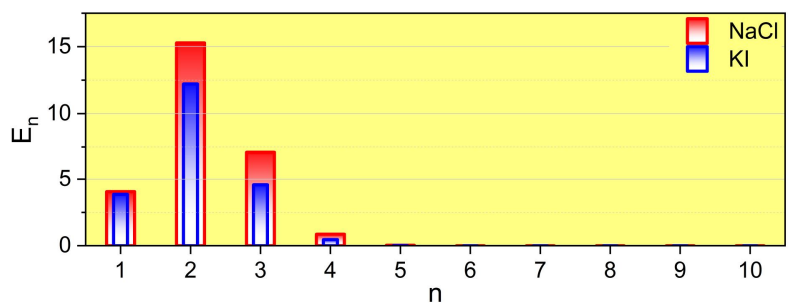

Fig. 5. Dependencies of the amplitude of the radial mode $E_{n}$ of the Cherenkov field on number $n, r_{L}=0.3 b$

And, eventually, at laser pulse radius increase to the value $r_{L}=0.9 b$ there is essential wakefield regularization. It is well visible in Fig. 6.

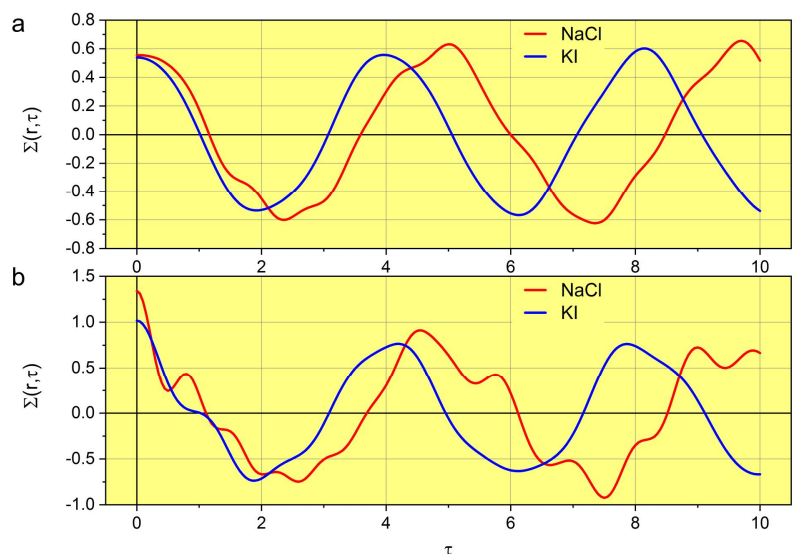

Fig. 6. Dependencies $\Sigma(r, \tau)$ for $r=0.4 b(a)$ and on the axis $r=0(b), r_{L}=0.9 b$ 


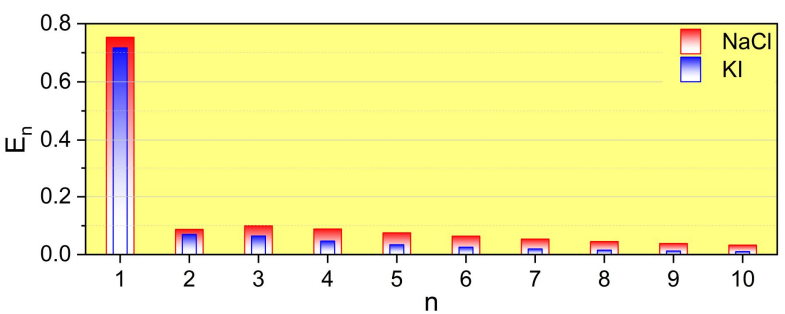

Fig. 7. Dependencies of amplitude of radial wakefield mode $E_{n}$ on number $n, r_{L}=0.9 b$

From Fig. 7 it follows that in this case of almost single mode operation of wakefield excitation is realized. The first (main) radial mode of ionic dielectric waveguide is excited mainly. Important circumstance is also that with increase in the transverse size of laser pulse as follows from the figures above, amplitudes of all excited radial modes of dielectric waveguide strongly decrease. This result is quite clear as at increase in laser radius $r_{L}$ degree of coherence of wakefield excitation by laser pulse strongly deteriorates.

It should be noted that similar change of picture of wakefield excitation by laser pulse will take place also at increase of pulse duration for its fixed radius.

\section{CONCLUSIONS}

In this work process of excitation of Cherenkov radiation by laser pulse in ionic dielectric waveguide is investigated. For definiteness, a diatomic ionic crystal medium is considered.

It is shown that in the terahertz (infrared) frequency range the excited electric field consists of potential field of longitudinal optical phonons and a set of eigen electromagnetic waves of dielectric waveguide (polaritons). Dielectric permittivity in terahertz (infrared) frequency range in ionic dielectrics always exceeds dielectric permittivity in optical range. Therefore the Cerenkov radiation condition for laser pulse in ionic dielectrics is always satisfied.

The frequency spectrum and space-time structure of the Cherenkov electromagnetic field excited by laser pulse in ionic dielectric waveguide is determined. It is shown that in case of laser pulse of small radius multimode operation of excitation of Cherenkov electromagnetic radiation is realized. Distribution of wakefield along waveguide has complicated irregular character. With increase in laser pulse radius the picture of field becomes simpler and, eventually, single mode operation of excitation is established. At the same time Cherenkov field amplitude decreases.

\section{REFERENCES}

1. S.A. Akhmanov, V.A. Fold. Optics of femtosecond laser pulses. M.: "Nauka", 1988, 388 p.

2. V.L. Ginzburg, V.I. Tsytovich. Transition radiation and transition scattering. M.: "Nauka", 1964, 360 p.

3. V.A. Balakirev, I.N. Onishchenko. Cherenkov radiation of a laser pulse in a dielectric waveguide // Problems of Atomic Science and Technology. Series “Plasma Physics”. 2018, № 6, p. 147-151.

4. V.A. Balakirev, I.N. Onishchenko. Wakefield excitation by a laser pulse in a dielectric medium // Problems of Atomic Science and Technology. Series "Plasma Electronics and New Methods of Acceleration”. 2018, № 4, p. 76-82.

5. V.A. Balakirev, I.N. Onishchenko. Cherenkov radiation of a laser pulse in ion dielectrics // Problems of Atomic Science and Technology. Series "Plasma Electronics and New Methods of Acceleration". 2019, № 4, p. 39-47.

6. V.A. Balakirev, I.N. Onishchenko. Wakefield excitation by a short laser pulse in ion dielectrics. arxiv: 2002. 09335 [physics.acc-ph].

7. M. Born, H. Kun. Dynamic theory of crystal lattice. M.: "Foreign literature", 1958, 488 p.

8. J. Sleter. Dielectrics, semiconductors, metals. M.: "Mir", 1969, 647 p.

9. N. Ashcroft, N. Mermin. Solid State Physics. M.: "Mir", 1978, v. 2, 392 p.

Article received 16.06.2021

\section{ЧЕРЕНКОВСКОЕ ВОЗБУЖДЕНИЕ КИЛЬВАТЕРНЫХ ЭЛЕКТРОМАГНИТНЫХ ВОЛН ЛАЗЕРНЫМ ИМПУЛЬСОМ В ИОННОМ ДИЭЛЕКТРИКЕ}

\section{В.А. Балакирев, П.И. Марков, И.Н. Онищенко}

Исследован процесс возбуждения черенковского электромагнитного излучения лазерным импульсом в ионном диэлектрическом волноводе. Определены частотный спектр и модовый состав черенковского излучения. Получена и исследована пространственно-временная структура черенковского электромагнитного поля.

\section{ЧЕРЕНКОВСЬКЕ ЗБУДЖЕННЯ КІЛЬВАТЕРНИХ ЕЛЕКТРОМАГНІТНИХ ХВИЛЬ ЛАЗЕРНИМ ІМПУЛЬСОМ В ІОННОМУ ДІЕЛЕКТРИКУ}

\section{В.А. Балакірев, П.І. Марков, І.М. Оніщенко}

Досліджено процес збудження черенковського електромагнітного випромінювання лазерним імпульсом в іонному діелектричному хвилеводі. Визначено частотний спектр та модовий склад черенковського випромінювання. Отримана й досліджена просторово-часова структура черенковського електромагнітного поля. 